\title{
A note on degenerate poly-Bernoulli numbers and polynomials
}

Dae San Kim ${ }^{1 *}$ and Taekyun Kim²

"Correspondence:
dskim@sogang.ac.kr
${ }^{1}$ Department of Mathematics,
Sogang University, Seoul, 121-742,
Republic of Korea
Full list of author information is
available at the end of the article

available at the end of the article

\begin{abstract}
In this paper, we consider the degenerate poly-Bernoulli polynomials and present new and explicit formulas for computing them in terms of the degenerate Bernoulli polynomials and Stirling numbers of the second kind.
\end{abstract}

MSC: 11B68; 11B73; 11B83

Keywords: degenerate poly-Bernoulli polynomial; degenerate Bernoulli polynomial; Stirling number of the second kind

\section{Introduction}

For $\lambda \in \mathbb{C}$, Carlitz considered the degenerate Bernoulli polynomials given by the generating function

$$
\frac{t}{(1+\lambda t)^{\frac{1}{\lambda}}-1}(1+\lambda t)^{\frac{x}{\lambda}}=\sum_{n=0}^{\infty} \beta_{n}(x \mid \lambda) \frac{t^{n}}{n !} \quad(\text { see }[1-3]) .
$$

When $x=0, \beta_{n}(\lambda)=\beta_{n}(0 \mid \lambda)$ are called the degenerate Bernoulli numbers.

Thus, by (1.1), we get

$$
\beta_{n}(x \mid \lambda)=\sum_{l=0}^{n}\left(\begin{array}{l}
n \\
l
\end{array}\right) \beta_{l}(\lambda)(x \mid \lambda)_{n-l}
$$

where $(x \mid \lambda)_{n}=x(x-\lambda)(x-2 \lambda) \cdots(x-\lambda(n-1))$.

The classical polylogarithm function $\mathrm{Li}_{k}$ is

$$
\operatorname{Li}_{k}(x)=\sum_{n=1}^{\infty} \frac{x^{n}}{n^{k}} \quad(k \in \mathbb{Z} \text {; see [2, 4-11]). }
$$

From (1.1), we note that

$$
\begin{aligned}
& \sum_{n=0}^{\infty} \lim _{\lambda \rightarrow 0} \beta_{n}(x \mid \lambda) \frac{t^{n}}{n !} \\
& \quad=\lim _{\lambda \rightarrow 0} \frac{t}{(1+\lambda t)^{\frac{1}{\lambda}}-1}(1+\lambda t)^{\frac{x}{\lambda}}
\end{aligned}
$$

(c) $2015 \mathrm{Kim}$ and Kim. This article is distributed under the terms of the Creative Commons Attribution 4.0 International License (http://creativecommons.org/licenses/by/4.0/), which permits unrestricted use, distribution, and reproduction in any medium, provided you give appropriate credit to the original author(s) and the source, provide a link to the Creative Commons license, and indicate if changes were made. 


$$
\begin{aligned}
& =\frac{t}{e^{t}-1} e^{x t} \\
& =\sum_{n=0}^{\infty} B_{n}(x) \frac{t^{n}}{n !},
\end{aligned}
$$

where $B_{n}(x)$ are called the Bernoulli polynomials (see [1-27]).

Thus, by (1.4), we get

$$
\lim _{\lambda \rightarrow 0} \beta_{n}(x \mid \lambda)=B_{n}(x) \quad(n \geq 0) .
$$

In $[4,14]$, the poly-Bernoulli polynomials are given by

$$
\frac{\mathrm{Li}_{k}\left(1-e^{-t}\right)}{e^{t}-1} e^{x t}=\sum_{n=0}^{\infty} B_{n}^{(k)}(x) \frac{t^{n}}{n !}
$$

For $k=1$, we have

$$
\begin{aligned}
\frac{\mathrm{Li}_{1}\left(1-e^{-t}\right)}{e^{t}-1} e^{x t} & =\frac{t}{e^{t}-1} e^{x t} \\
& =\sum_{n=0}^{\infty} B_{n}(x) \frac{t^{n}}{n !} .
\end{aligned}
$$

By (1.4) and (1.7), we get $B_{n}^{(1)}(x)=B_{n}(x)$.

The Stirling numbers of the second kind are given by

$$
x^{n}=\sum_{l=0}^{n} S_{2}(n, l)(x)_{l} \quad(\text { see }[1-27]),
$$

and the Stirling numbers of the first kind are defined by

$$
(x)_{n}=x(x-1) \cdots(x-n+1)=\sum_{l=0}^{n} S_{1}(n, l) x^{l} \quad(n \geq 0) .
$$

The purpose of this paper is to construct the degenerate poly-Bernoulli polynomials and present new and explicit formulas for computing them in terms of the degenerate Bernoulli polynomials and Stirling numbers of the second kind.

\section{Degenerate poly-Bernoulli numbers and polynomials}

For $\lambda \in \mathbb{C}, k \in \mathbb{Z}$, we consider the degenerate poly-Bernoulli polynomials given by the generating function

$$
\frac{\mathrm{Li}_{k}\left(1-e^{-t}\right)}{(1+\lambda t)^{\frac{1}{\lambda}}-1}(1+\lambda t)^{\frac{x}{\lambda}}=\sum_{n=0}^{\infty} \beta_{n}^{(k)}(x \mid \lambda) \frac{t^{n}}{n !} .
$$

When $x=0, \beta_{n}^{(k)}(\lambda)=\beta_{n}^{(k)}(0 \mid \lambda)$ are called the degenerate poly-Bernoulli numbers. Note that $\beta_{n}^{(1)}(x \mid \lambda)=\beta_{n}(x \mid \lambda)$ and $\lim _{\lambda \rightarrow 0} \beta_{n}^{(k)}(x \mid \lambda)=B_{n}^{(k)}(x)$. 
From (2.1), we can derive the following equation:

$$
\begin{aligned}
\sum_{n=0}^{\infty} \beta_{n}^{(k)}(x \mid \lambda) \frac{t^{n}}{n !} & =\left(\frac{\operatorname{Li}_{k}\left(1-e^{-t}\right)}{(1+\lambda t)^{\frac{1}{\lambda}}-1}\right)(1+\lambda t)^{\frac{x}{\lambda}} \\
& =\left(\sum_{l=0}^{\infty} \beta_{l}^{(k)}(\lambda) \frac{t^{l}}{l !}\right)\left(\sum_{m=0}^{\infty}(x \mid \lambda)_{m} \frac{t^{m}}{m !}\right) \\
& =\sum_{n=0}^{\infty}\left(\sum_{l=0}^{n}\left(\begin{array}{l}
n \\
l
\end{array}\right) \beta_{l}^{(k)}(\lambda)(x \mid \lambda)_{n-l}\right) \frac{t^{n}}{n !} .
\end{aligned}
$$

Thus, by (2.2), we get

$$
\beta_{n}^{(k)}(x \mid \lambda)=\sum_{l=0}^{n}\left(\begin{array}{l}
n \\
l
\end{array}\right) \beta_{l}^{(k)}(\lambda)(x \mid \lambda)_{n-l}
$$

Now, we observe that

$$
\begin{aligned}
& \frac{\operatorname{Li}_{k}\left(1-e^{-t}\right)}{(1+\lambda t)^{\frac{1}{\lambda}}-1}(1+t)^{\frac{x}{\lambda}} \\
& \quad=\sum_{n=0}^{\infty} \beta_{n}^{(k)}(x \mid \lambda) \frac{t^{n}}{n !} \\
& \quad=\frac{(1+t)^{\frac{x}{\lambda}}}{(1+\lambda t)^{\frac{1}{\lambda}}-1} \int_{0}^{t} \underbrace{\frac{1}{e^{y}-1} \int_{0}^{y} \frac{1}{e^{y}-1} \int_{0}^{y} \cdots \frac{1}{e^{y}-1} \int_{0}^{y} \frac{y}{e^{y}-1} d y \cdots d y .}_{(k-2) \text { times }}
\end{aligned}
$$

From (2.4), we have

$$
\begin{aligned}
& \sum_{n=0}^{\infty} \beta_{n}^{(2)}(x \mid \lambda) \frac{t^{n}}{n !} \\
& \quad=\frac{(1+t)^{\frac{x}{\lambda}}}{(1+\lambda t)^{\frac{1}{\lambda}}-1} \int_{0}^{t} \frac{y}{e^{y}-1} d y \\
& =\frac{(1+t)^{\frac{x}{\lambda}}}{(1+\lambda t)^{\frac{1}{\lambda}}-1} \sum_{l=0}^{\infty} \frac{B_{l}}{l !} \int_{0}^{t} y^{l} d y \\
& =\left(\frac{t}{(1+\lambda t)^{\frac{1}{\lambda}}-1}(1+\lambda t)^{\frac{x}{\lambda}}\right)\left(\sum_{l=0}^{\infty} \frac{B_{l}}{l+1} \frac{t^{l}}{l !}\right) \\
& =\sum_{n=0}^{\infty}\left\{\sum_{l=0}^{n}\left(\begin{array}{l}
n \\
l
\end{array}\right) \frac{B_{l}}{l+1} \beta_{n-l}(x \mid \lambda)\right\} \frac{t^{n}}{n !},
\end{aligned}
$$

where $B_{n}=B_{n}(0)$ are the Bernoulli numbers.

By comparing the coefficients on both sides of (2.5), we obtain the following theorem. 
Theorem 2.1 For $n \geq 0$, we have

$$
\begin{aligned}
\beta_{n}^{(2)}(x \mid \lambda) & =\sum_{l=0}^{n}\left(\begin{array}{l}
n \\
l
\end{array}\right) \frac{B_{l}}{l+1} \beta_{n-l}(x \mid \lambda) \\
& =\beta_{n}(x \mid \lambda)-\frac{n}{4} \beta_{n-1}(x \mid \lambda)+\sum_{l=2}^{n}\left(\begin{array}{l}
n \\
l
\end{array}\right) \frac{B_{l}}{l+1} \beta_{n-l}(x \mid \lambda) .
\end{aligned}
$$

Moreover,

$$
\beta_{n}^{(k)}(x \mid \lambda)=\sum_{l=0}^{n}\left(\begin{array}{l}
n \\
l
\end{array}\right) \beta_{l}^{(k)}(\lambda)(x \mid \lambda)_{n-l}
$$

By (2.4), we easily get

$$
\begin{aligned}
& \sum_{n=0}^{\infty} \beta_{n}^{(k)}(x \mid \lambda) \frac{t^{n}}{n !} \\
& \quad=\frac{\operatorname{Li}_{k}\left(1-e^{-t}\right)}{(1+\lambda t)^{\frac{1}{\lambda}}-1}(1+t)^{\frac{x}{\lambda}} \\
& =\frac{t}{(1+\lambda t)^{\frac{1}{\lambda}}-1}(1+t)^{\frac{x}{\lambda}} \frac{\operatorname{Li}_{k}\left(1-e^{-t}\right)}{t} .
\end{aligned}
$$

We observe that

$$
\begin{aligned}
\frac{1}{t} \operatorname{Li}_{k}\left(1-e^{-t}\right) & =\frac{1}{t} \sum_{n=1}^{\infty} \frac{1}{n^{k}}\left(1-e^{-t}\right)^{n} \\
& =\frac{1}{t} \sum_{n=1}^{\infty} \frac{(-1)^{n}}{n^{k}} n ! \sum_{l=n}^{\infty} S_{2}(l, n) \frac{(-t)^{l}}{l !} \\
& =\frac{1}{t} \sum_{l=1}^{\infty} \sum_{n=1}^{l} \frac{(-1)^{n+l}}{n^{k}} n ! S_{2}(l, n) \frac{t^{l}}{l !} \\
& =\sum_{l=0}^{\infty} \sum_{n=1}^{l+1} \frac{(-1)^{n+l+1}}{n^{k}} n ! \frac{S_{2}(l+1, n)}{l+1} \frac{t^{l}}{l !} .
\end{aligned}
$$

From (2.6) and (2.7), we have

$$
\begin{aligned}
& \sum_{n=0}^{\infty} \beta_{n}^{(k)}(x \mid \lambda) \frac{t^{n}}{n !} \\
& \quad=\left(\sum_{m=0}^{\infty} \beta_{m}(x \mid \lambda) \frac{t^{m}}{m !}\right)\left(\sum_{l=0}^{\infty}\left(\sum_{p=1}^{l+1} \frac{(-1)^{p+l+1}}{p^{k}} p ! \frac{S_{2}(l+1, p)}{l+1}\right) \frac{t^{l}}{l !}\right) \\
& \quad=\sum_{n=0}^{\infty}\left\{\sum_{l=0}^{n}\left(\begin{array}{l}
n \\
l
\end{array}\right)\left(\sum_{p=1}^{l+1} \frac{(-1)^{p+l+1} p !}{p^{k}} \frac{S_{2}(l+1, p)}{l+1}\right) \beta_{n-l}(x \mid \lambda)\right\} \frac{t^{n}}{n !} .
\end{aligned}
$$

By comparing the coefficients on both sides of (2.8), we obtain the following theorem. 
Theorem 2.2 For $n \geq 0$, we have

$$
\beta_{n}^{(k)}(x \mid \lambda)=\sum_{l=0}^{n}\left(\begin{array}{l}
n \\
l
\end{array}\right)\left(\sum_{p=1}^{l+1} \frac{(-1)^{p+l+1} p !}{p^{k}} \frac{S_{2}(l+1, p)}{l+1}\right) \beta_{n-l}(x \mid \lambda) .
$$

It is easy to show that

$$
\begin{aligned}
& \frac{\operatorname{Li}_{k}\left(1-e^{-t}\right)}{(1+\lambda t)^{\frac{1}{\lambda}}-1}(1+\lambda t)^{\frac{x+1}{\lambda}}-\frac{\operatorname{Li}_{k}\left(1-e^{-t}\right)}{(1+\lambda t)^{\frac{1}{\lambda}}-1}(1+\lambda t)^{\frac{x}{\lambda}} \\
& =(1+\lambda t)^{\frac{x}{\lambda}} \operatorname{Li}_{k}\left(1-e^{-t}\right) \\
& =\left(\sum_{l=0}^{\infty}(x \mid \lambda)_{l} \frac{t^{l}}{l !}\right)\left(\sum_{m=1}^{\infty} \frac{\left(1-e^{-t}\right)^{m}}{m^{k}}\right) \\
& =\left(\sum_{l=0}^{\infty}(x \mid \lambda)_{l} \frac{t^{l}}{l !}\right)\left(\sum_{m=0}^{\infty} \frac{\left(1-e^{-t}\right)^{m+1}}{(m+1)^{k}}\right) \\
& =\left(\sum_{l=0}^{\infty}(x \mid \lambda)_{l} \frac{t^{l}}{l !}\right)\left(\sum_{p=1}^{\infty}\left(\sum_{m=0}^{p-1} \frac{(-1)^{m+p+1}}{(m+1)^{k}}(m+1) ! S_{2}(p, m+1)\right) \frac{t^{p}}{p !}\right) \\
& \quad=\sum_{n=1}^{\infty}\left\{\sum_{p=1}^{n} \sum_{m=0}^{p-1} \frac{(-1)^{m+p+1}}{(m+1)^{k}}(m+1) ! S_{2}(p, m+1)\left(\begin{array}{c}
n \\
p
\end{array}\right)(x \mid \lambda)_{n-p}\right\} \frac{t^{n}}{n !} .
\end{aligned}
$$

On the other hand,

$$
\begin{gathered}
\frac{\operatorname{Li}_{k}\left(1-e^{-t}\right)}{(1+\lambda t)^{\frac{1}{\lambda}}-1}(1+\lambda t)^{\frac{x+1}{\lambda}}-\frac{\operatorname{Li}_{k}\left(1-e^{-t}\right)}{(1+\lambda t)^{\frac{1}{\lambda}}-1}(1+\lambda t)^{\frac{x}{\lambda}} \\
=\sum_{n=0}^{\infty}\left\{\beta_{n}^{(k)}(x+1 \mid \lambda)-\beta_{n}^{(k)}(x \mid \lambda)\right\} \frac{t^{n}}{n !} .
\end{gathered}
$$

Therefore, by (2.9) and (2.10), we obtain the following theorem.

Theorem 2.3 For $n \geq 1$, we have

$$
\begin{aligned}
& \beta_{n}^{(k)}(x+1 \mid \lambda)-\beta_{n}^{(k)}(x \mid \lambda) \\
& =\sum_{p=1}^{n}\left(\sum_{m=0}^{p-1} \frac{(-1)^{m+k+1}}{(m+1)^{k}}(m+1) ! S_{2}(k+m+1)\right)\left(\begin{array}{l}
n \\
p
\end{array}\right)(x \mid \lambda)_{n-p} .
\end{aligned}
$$

Now, we note that

$$
\begin{aligned}
& \frac{\operatorname{Li}_{k}\left(1-e^{-t}\right)}{(1+\lambda t)^{\frac{1}{\lambda}}-1}(1+\lambda t)^{\frac{x}{\lambda}} \\
& =\frac{\operatorname{Li}_{k}\left(1-e^{-t}\right)}{(1+\lambda t)^{\frac{d}{\lambda}}-1} \sum_{a=0}^{d-1}(1+\lambda t)^{\frac{l+x}{\lambda}} \\
& =\left(\frac{\operatorname{Li}_{k}\left(1-e^{-t}\right)}{t}\right) \frac{1}{d} \sum_{a=0}^{d-1} \frac{d t}{(1+\lambda t)^{\frac{d}{\lambda}}-1}(1+\lambda t)^{\frac{l+x}{\lambda}}
\end{aligned}
$$




$$
\begin{aligned}
= & \sum_{l=0}^{\infty}\left(\sum_{p=1}^{l+1} \frac{(-1)^{p+l+1}}{p^{k}} p ! \frac{S_{2}(l+1, p)}{l+1}\right) \frac{t^{l}}{l !} \\
& \times \sum_{a=0}^{d-1} \sum_{m=0}^{\infty} \beta_{m}\left(\frac{l+x}{d} \mid \frac{\lambda}{d}\right) d^{m-1} \frac{t^{m}}{m !} \\
= & \sum_{a=0}^{d-1}\left(\sum_{n=0}^{\infty}\left(\sum_{l=0}^{n} \sum_{p=1}^{l+1}\left(\begin{array}{l}
n \\
l
\end{array}\right) \frac{(-1)^{p+l+1}}{p^{k}} p ! \frac{S_{2}(l+1, p)}{l+1} \beta_{n-l}\left(\frac{l+x}{d} \mid \frac{\lambda}{d}\right) d^{n-l-1}\right) \frac{t^{n}}{n !}\right) \\
= & \sum_{n=0}^{\infty}\left\{\sum_{a=0}^{d-1} \sum_{l=0}^{n} \sum_{p=1}^{l+1}\left(\begin{array}{l}
n \\
l
\end{array}\right) \frac{(-1)^{p+l+1}}{p^{k}} p ! \frac{S_{2}(l+1, p)}{l+1} \beta_{n-l}\left(\frac{l+x}{d} \mid \frac{\lambda}{d}\right) d^{n-l-1}\right\} \frac{t^{n}}{n !},
\end{aligned}
$$

where $d$ is a fixed positive integer.

On the other hand,

$$
\begin{gathered}
\frac{\operatorname{Li}_{k}\left(1-e^{-t}\right)}{(1+\lambda t)^{\frac{1}{\lambda}}-1}(1+\lambda t)^{\frac{x}{\lambda}} \\
\quad=\sum_{n=0}^{\infty} \beta_{n}^{(k)}(x \mid \lambda) \frac{t^{n}}{n !} .
\end{gathered}
$$

Therefore, by (2.11) and (2.12), we obtain the following theorem.

Theorem 2.4 For $n \geq 0, d \in \mathbb{N}$ and $k \in \mathbb{Z}$, we have

$$
\begin{aligned}
& \beta_{n}^{(k)}(x \mid \lambda) \\
& =\sum_{a=0}^{d-1} \sum_{l=0}^{n} \sum_{p=1}^{l+1}\left(\begin{array}{l}
n \\
l
\end{array}\right) \frac{(-1)^{p+l+1}}{p^{k}} p ! \frac{S_{2}(l+1, p)}{l+1} \beta_{n-l}\left(\frac{l+x}{d} \mid \frac{\lambda}{d}\right) d^{n-l-1} .
\end{aligned}
$$

From (2.4), we can derive the following equation:

$$
\begin{aligned}
& \sum_{n=0}^{\infty} \beta_{n}^{(k)}(x+y \mid \lambda) \frac{t^{n}}{n !} \\
& =\frac{\operatorname{Li}_{k}\left(1-e^{-t}\right)}{(1+\lambda t)^{\frac{1}{\lambda}}-1}(1+\lambda t)^{\frac{x+y}{\lambda}} \\
& =\left(\frac{\operatorname{Li}_{k}\left(1-e^{-t}\right)}{(1+\lambda t)^{\frac{1}{\lambda}}-1}(1+t \lambda)^{\frac{x}{\lambda}}\right)(1+\lambda t)^{\frac{y}{\lambda}} \\
& =\left(\sum_{l=0}^{\infty} \beta_{l}^{(k)}(x \mid \lambda) \frac{t^{l}}{l !}\right)\left(\sum_{m=0}^{\infty}(y \mid \lambda)_{m} \frac{t^{m}}{m !}\right) \\
& =\sum_{n=0}^{\infty}\left(\sum_{l=0}^{n}\left(\begin{array}{l}
n \\
l
\end{array}\right) \beta_{l}^{(k)}(x \mid \lambda)(y \mid \lambda)_{n-l}\right) \frac{t^{n}}{n !} .
\end{aligned}
$$

Therefore, by (2.13), we obtain the following theorem. 
Theorem 2.5 For $n \geq 0$, we have

$$
\beta_{n}^{(k)}(x+y \mid \lambda)=\sum_{l=0}^{n}\left(\begin{array}{l}
n \\
l
\end{array}\right) \beta_{l}^{(k)}(x \mid \lambda)(y \mid \lambda)_{n-l}
$$

\section{Remark}

$$
\begin{aligned}
& \frac{d}{d x} \beta_{n}^{(k)}(x \mid \lambda) \\
& \quad=\frac{d}{d x} \sum_{l=0}^{n}\left(\begin{array}{l}
n \\
l
\end{array}\right) \beta_{n-l}^{(k)}(\lambda)(x \mid \lambda)_{l} \\
& \quad=\sum_{l=0}^{n}\left(\begin{array}{l}
n \\
l
\end{array}\right) \beta_{n-l}^{(k)}(\lambda) \sum_{j=0}^{l-1} \frac{1}{x-\lambda j} \prod_{i=0}^{l-1}(x-\lambda i) \\
& =\sum_{l=0}^{n}\left(\begin{array}{l}
n \\
l
\end{array}\right) \beta_{n-l}^{(k)}(\lambda) \sum_{j=0}^{l-1} \prod_{\substack{i=0 \\
i \neq j}}^{l-1}(x-\lambda i) .
\end{aligned}
$$

\section{Competing interests}

The authors declare that they have no competing interests.

\section{Authors' contributions}

All authors contributed equally to this work. All authors read and approved the final manuscript.

\section{Author details}

${ }^{1}$ Department of Mathematics, Sogang University, Seoul, 121-742, Republic of Korea. ${ }^{2}$ Department of Mathematics, Kwangwoon University, Seoul, 139-701, Republic of Korea.

\section{Acknowledgements}

The authors would like to thank the referees for their valuable comments.

\section{Received: 18 March 2015 Accepted: 6 August 2015 Published online: 20 August 2015}

\section{References}

1. Carlitz, L: Degenerate Stirling, Bernoulli and Eulerian numbers. Util. Math. 15, 51-88 (1979)

2. Kim, DS, Kim, T, Dolgy, DV, Komatsu, T: Barnes-type degenerate Bernoulli polynomials. Adv. Stud. Contemp. Math. 25(1), 121-146 (2015)

3. Kim, T: Barnes' type multiple degenerate Bernoulli and Euler polynomials. Appl. Math. Comput. 258, 556-564 (2015)

4. Jolany, H, Mohebbi, H: Some results on Generalized multi poly-Bernoulli and Euler polynomials. Int. J. Math. Comb. 2, 117-129 (2011)

5. Kim, DS, Kim, T, Mansour, T, Dolgy, DV: On poly-Bernoulli polynomials of the second kind with umbral calculus viewpoint. Adv. Differ. Equ. 2015(1), 27 (2015)

6. Kim, DS, Kim, T: Some identities of degenerate Euler polynomials arising from $p$-adic fermionic integrals on $\mathbb{Z}_{p}$. Integral Transforms Spec. Funct. 26(4), 295-302 (2015)

7. Kim, DS, Kim, T: Higher-order Frobenius-Euler and poly-Bernoulli mixed-type polynomials. Adv. Differ. Equ. 2013, 251 (2013)

8. Kim, DS, Kim, T: Hermite and poly-Bernoulli mixed-type polynomials. Adv. Differ. Equ. 2013(343), 12 (2013)

9. Kim, DS, Kim, T: A note on poly-Bernoulli and higher-order poly-Bernoulli polynomials. Russ. J. Math. Phys. 22(1), 26-33 (2015)

10. Kim, DS, Kim, T, Lee, SH: A note on poly-Bernoulli polynomials arising umbral calculus. Adv. Stud. Theor. Phys. 7(15), 731-744 (2013)

11. Kim, T, Jang, YS, Seo, JJ: Poly-Bernoulli polynomials and their applications. Int. J. Math. Anal. 8(30), 1495-1503 (2014)

12. Acikgoz, M, Erdal, D, Araci, S: A new approach to $q$-Bernoulli numbers and $q$-Bernoulli polynomials related to q-Bernstein polynomials. Adv. Differ. Equ. 9, Article ID 951764 (2010)

13. Araci, S, Acikgoz, M, Jolany, H: On the families of $q$-Euler polynomials and their applications. J. Egypt. Math. Soc. 23(1), 1-5 (2015)

14. Bayad, A, Hamahata, Y: Multiple polylogarithms and multi-poly-Bernoulli polynomials. Funct. Approx. Comment. Math. 46, 45-61 (2012)

15. Bayad, A, Gaboury, S: Generalized Dirichlet L-function of arbitrary order with applications. Adv. Stud. Contemp. Math. (Kyungshang) 23(4), 607-619 (2013)

16. Gaboury, S, Tremblay, R, Fugère, B-J: Some explicit formulas for certain new classes of Bernoulli, Euler and Genocchi polynomials. Proc. Jangjeon Math. Soc. 17(1), 115-123 (2014) 
17. Carlitz, L: A degenerate Staudt-Clausen theorem. Arch. Math. (Basel) 7, 28-33 (1956)

18. Dere, R, Simsek, Y: Applications of umbral algebra to some special polynomials. Adv. Stud. Contemp. Math. (Kyungshang) 22(3), 433-438 (2012)

19. Ding, D, Yang, J: Some identities related to the Apostol-Euler and Apostol-Bernoulli polynomials. Adv. Stud. Contemp. Math. (Kyungshang) 20(1), 7-21 (2010)

20. Kim, T, Kwon, HK, Lee, SH, Seo, JJ: A note on poly-Bernoulli numbers and polynomials of the second kind. Adv. Differ Equ. 2014, 219 (2014)

21. Kim, T: Some identities on the $q$-Euler polynomials of higher order and $q$-Stirling numbers by the fermionic $p$-adic integral on $\mathbb{Z}_{p}$. Russ. J. Math. Phys. 16(4), 484-491 (2009)

22. Luo, Q-M, Guo, B-N, Qi, F: On evaluation of Riemann zeta function $\zeta$ (s). Adv. Stud. Contemp. Math. (Kyungshang) 7(2) 135-144 (2003)

23. Luo, Q-M, Qi, F: Relationships between generalized Bernoulli numbers and polynomials and generalized Euler numbers and polynomials. Adv. Stud. Contemp. Math. (Kyungshang) 7(1), 11-18 (2003)

24. Park, J-W, Rim, S-H: On the modified q-Bernoulli polynomials with weight. Proc. Jangjeon Math. Soc. 17(2), 231-236 (2014)

25. Şen, E: Theorems on Apostol-Euler polynomials of higher order arising from Euler basis. Adv. Stud. Contemp. Math. (Kyungshang) 23(2), 337-345 (2013)

26. Srivastava, HM, Kim, T, Simsek, Y: $q$-Bernoulli numbers and polynomials associated with multiple $q$-zeta functions and basic L-series. Russ. J. Math. Phys. 12(2), 241-268 (2005)

27. Zhang, Z, Yang, J: On sums of products of the degenerate Bernoulli numbers. Integral Transforms Spec. Funct. 20(9-10), 751-755 (2009)

\section{Submit your manuscript to a SpringerOpen ${ }^{\circ}$ journal and benefit from:}

- Convenient online submission

- Rigorous peer review

- Immediate publication on acceptance

- Open access: articles freely available online

- High visibility within the field

- Retaining the copyright to your article 